\title{
Trends in psychotropic prescribing in general practice and general medical patients
}

\author{
Allan R. Smith ${ }^{1}$, Morag McIntosh ${ }^{2}$, Gordon T. McInnes ${ }^{3}$ and David H. Lawson ${ }^{4}$ \\ ${ }^{1}$ Department of Pharmacy, Knightswood Hospital, Glasgow G13 $2 X G,{ }^{2}$ Department of Pharmacy, Western \\ Infirmary, Glasgow G11 6NT, ${ }^{3}$ University Department of Medicine, Western Infirmary, Glasgow G11 6NT, and \\ ${ }^{4}$ Department of Clinical Pharmacology, Royal Infirmary, Glasgow G4 OSF, UK.
}

\begin{abstract}
Summary: Psychotropic drug prescribing in 1280 medical inpatients between 1973-75 was compared with that in 1200 similar patients during 1982-83. Three benzodiazepines accounted for $64 \%$ of prescriptions in 1973-75 and nine benzodiazepines for 82\% of prescriptions in 1982-83. Over the decade, use of psychotropic drugs fell from $56 \%$ to $38 \%(P<0.001)$, primarily due to a reduction in patients treated only in hospital $(34 \%$ vs $16 \%, P<0.001)$. Before admission, the proportions of patients receiving these drugs were similar (17\% vs 18\%). During admission, concomitant administration of similar drugs declined from $22 \%$ of patients in $1973-75$ to $11 \%$ in $1982-83(P<0.001)$, while concurrent prescribing before admission increased from 13 to $19 \%$. The marked fall in psychotropic drug use and in inappropriate concomitant therapy indicates an encouraging trend towards more rational drug use at least in hospital. This was achieved without fiscal control and further rationalization of prescribing habits may be achieved by self-audit within the profession without legislative action.
\end{abstract}

\section{Introduction}

In the last decade the number of psychotropic drugs available to the prescriber has increased dramatically. In particular, many new benzodiazepines have been marketed and barbiturates are now considered inappropriate for use as routine hypnotics and tranquillizers (Committee on the Review of Medicines, 1979). The pharmacokinetic, physicochemical and pharmacodynamic properties of benzodiazepines differ (Greenblatt et al., 1981), yet current evidence suggests the therapeutic response to these drugs is broadly similar (Greenblatt \& Shader, 1974; Kesson et al., 1976). Claims of particular advantages for individual drugs have encouraged prescribers to reserve certain preparations for specific indications, a policy which often results in patients receiving more than one pharmacologically similar entity. It is unlikely that this represents optimal practice, since it may facilitate toxicity, is inconvenient to the patient and is expensive.

In order to explore the effect on prescribing of the large number of new compounds appearing on the market in the 1970s we have reviewed details of the use

Correspondence: G.T. McInnes, B.Sc., M.D., M.R.C.P. Accepted: 6 February 1986 of psychotropic drugs in medical inpatients admitted to a Scottish teaching hospital between 1973 and 1975 and compared them with similar information acquired between 1982 and 1983. Our objectives were to investigate changes in prescribing habits with particular reference to the extent of concomitant prescriptions of psychotropic drugs and the influence of hospital admission on the use of these drugs in the community.

\section{Materials and methods}

A total of 2480 patients admitted to general medical wards of the Western Infirmary, Glasgow, were reviewed. Between 1973 and 1975, information was gathered on 1280 consecutive inpatients as part of the Boston Collaborative Drug Surveillance Program. The methods employed have been described in detail elsewhere (Jick et al., 1970). The data gathered included the patient's name and address, diagnosis, date of admission and discharge and the outcome of hospitalization. The prescription of psychotropic drugs before and during admission and on discharge was recorded and information on indication, dosage 
Table I Psychotropic drug use in hospitalized patients

\begin{tabular}{lccc}
\hline & $1973-75$ & $1982-83$ & \\
\hline $\begin{array}{l}\text { Patients receiving psy- } \\
\text { chotropic drugs during survey }\end{array}$ & $712(56 \%)$ & $455(38 \%)$ & $\chi^{2}=92.7$ \\
$\begin{array}{l}\text { Patients receiving psy- } \\
\text { chotropic drugs before admis- } \\
\text { sion }\end{array}$ & $220(17 \%)$ & $213(18 \%)$ & NS \\
$\begin{array}{l}\text { Patients receiving psy- } \\
\text { chotropic drugs in hospital }\end{array}$ & $641(50 \%)$ & $391(33 \%)$ & $\chi^{2}=78.1$ \\
$\begin{array}{l}\text { Patients receiving psy- } \\
\text { chotropic drugs on discharge }\end{array}$ & $98(7.7 \%)$ & $111(9.3 \%)$ & $P<0.001$ \\
\begin{tabular}{l} 
No. of patients \\
\hline
\end{tabular} & $1280(100 \%)$ & $1200(100 \%)$ & NS \\
\hline
\end{tabular}

NS $=$ not significant

and frequency of administration of the drug was also noted. Each patient was admitted to the survey only once. During 1982 and 1983 information on 1200 consecutive inpatients in the same wards was collected in a similar manner using standardized self-coding sheets.

Psychotropic drugs were defined as those hypnotics, sedatives, anxiolytics, antipsychotic drugs and antidepressants included in the British National Formulary Chapter 4, Sections 1-3. Thus, central nervous stimulants, appetite suppressants, drugs used in nausea and vertigo, analgesics, anti-epileptics and drugs used in Parkinsonism and related disorders were excluded from the review. Concomitant psychotropic drug prescribing was defined as occurring when a patient was prescribed two or more psychotropic drugs simultaneously for longer than one day.

The average age of the patients was 58 years and $48 \%$ were male. The demographic characteristics during the two periods of study were comparable: 1973-75, mean age 58 years (range 12-92) 49\% male;
1982-83, mean age 58 years (range 13-96) $46 \%$ male. The distribution of discharge diagnoses was similar in the two groups of patients $-34 \%$ had cardiovascular disease, $14 \%$ respiratory disease, $7 \%$ haematological disease, $6 \%$ endocrine disorders, $5 \%$ gastrointestinal diseases, $5 \%$ tumours and $32 \%$ other disorders of undiagnosed symptomatology (International Classification of Disease, 9th revision).

\section{Results}

There was a marked fall in overall prescriptions of psychoactive drugs between the two study periods. Of the 1280 patients assessed between 1973 and 1975 , $56 \%$ were prescribed psychotropic drugs compared with $38 \%$ of the 1200 patients surveyed between 1982 and $1983\left(\chi^{2}=92.7, P<0.001\right)$. This difference was due primarily to a substantial fall in the proportion of patients receiving psychotropic drugs only during admission to hospital - $435(34 \%)$ in $1973-75$ com-

Table II Concomitant psychoactive drug therapy in hospitalized patients

\begin{tabular}{lccccccccc}
\hline & \multicolumn{3}{c}{$1973-75$} & \multicolumn{3}{c}{$1982-83$} \\
& Number & Total & $\%$ & Number & Total & $\%$ & \\
& 28 & 220 & 13 & 40 & 213 & 19 & NS \\
Before admission & 144 & 641 & 22 & 42 & 390 & 11 & $\chi^{2}=21.6$ \\
During admission & 14 & 98 & 14 & 11 & 111 & 9.9 & NS \\
On discharge & & & & & & & \\
\hline
\end{tabular}

NS = not significant 


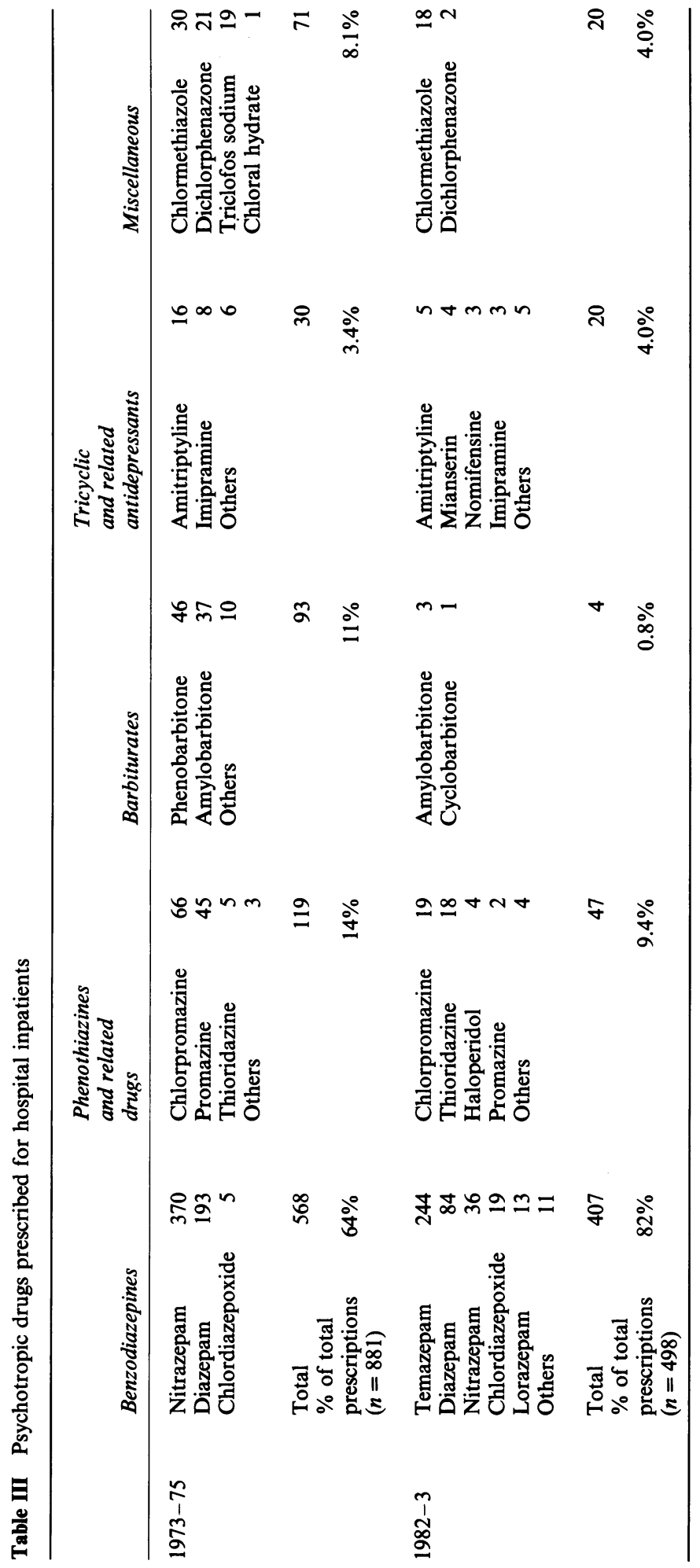


pared with $196(16 \%)$ during 1982-83. By contrast, the proportions of patients receiving these drugs before admission were similar in the two periods $(17 \%$ in 1973-75 and 18\% in 1982-83). Details of the frequency of psychoactive drug use are shown in Table I.

Concomitant prescribing of two or more psychotropic drugs occurred during admission in 144 of the 641 patients $(22 \%)$ receiving psychotropic drugs in 1973-75 compared with 42 of the 390 recipients $(11 \%)$ in $1982-83\left(\chi^{2}=21.6, P<0.001\right)$. Thus in $1982-83$ not only was there a substantial fall in the first use of psychoactive drugs in hospital compared with the earlier period but there was also a threefold reduction in the actual numbers of patients receiving multiple psychoactive drugs concurrently (Table II).

The psychotropic drugs used during admission to hospital are shown in Table III. In both surveys, benzodiazepines were the drugs prescribed most frequently, accounting for $64 \%$ of 881 prescriptions between $1973-75$ and for $82 \%$ of 498 prescriptions during 1982-83. In the intervening years the number of different benzodiazepines prescribed increased from three to nine. Barbiturate use declined from $11 \%$ to $0.8 \%$.

In both periods, the majority of prescriptions were written to treat insomnia (62\% of prescriptions, $35 \%$ of patients), while the second most frequent indication was sedation/relief of anxiety ( $31 \%$ of prescriptions, $17 \%$ of patients). Only a small minority of prescriptions were used ostensibly to treat diagnosed psychosis (4.5\% in $1973-75$ and $2.2 \%$ in $1982-83$ ) but major tranquillizers such as phenothiazines accounted for $14 \%$ of prescriptions in $1973-75$ and $9.4 \%$ in 1982-83. These drugs were often used as sedatives in patients with alcoholism or severe respiratory disease. The proportion of prescriptions for the treatment of depression $(3.3 \%)$ was similar in each time period.

\section{Discussion}

Well over one half $(56 \%)$ of all patients admitted to general medical wards of a Scottish teaching hospital in 1973-75 had received psychotropic drugs whereas this frequency had fallen to $38 \%$ in the period 1982-83. One customary explanation for the frequent use of psychoactive drugs in hospitalized patients is the treatment of apprehension at the prospect of hospitalization. However this is an unlikely explanation since some $17 \%$ of patients were receiving psychotropic drugs before admission, a frequency only slightly greater than that generally quoted for such prescribing in the community (Rickels, 1981). Moreover, the willingness of hospital physicians to discontinue therapy in many patients upon admission suggests that these influences do not explain the prescribing habits. The substantial decrease in prescribing over the decade suggests that hospital doctors have modified their view of anxiety from an illness requiring drug treatment to an unavoidable part of everyday life and also became more aware of the adverse effects of psychotropic drugs, particularly in the elderly (Thompson et al., 1983). During the same time interval, primary care physicians appear to have altered their prescribing only marginally.

The major explanation for the observed fall in use of psychoactive drugs between the two study periods was the marked fall in first time prescriptions in hospitals. In both periods hospital physicians tended to discontinue psychotropic drugs prescribed before admission, a fact which may reflect an improvement in the patient's clinical state or may represent a more critical assessment of the need for such drugs. Although hospital physicians were not reluctant to use psychotropic drugs, in general they did not continue these prescriptions on discharge.

The number of different benzodiazepines in use in hospital increased from three to nine between 1973 and 1983. Although the newer benzodiazepines may have improved effectiveness and fewer side effects it is doubtful whether one hospital requires to have as many different benzodiazepines in use. Indeed, since this survey hospital physicians have been active in reducing the number of medicines available for routine use by the introduction of hospital formularies (Barret et al., 1982), and more recently the Government has introduced a Limited List primarily to extend this concept into the community.

Concomitant prescribing of psychotropic drugs in hospital has fallen by half between 1974 and 1983. Over the same period simultaneous prescribing before admission rose from $13 \%$ to $19 \%$ although because of small numbers, this difference could have arisen by chance. Concomitant administration of these agents cannot generally be considered rational, particularly when it involves two different benzodiazepines (Kesson et al., 1976).

Comparison of hospital prescribing in the two survey periods is of particular interest at a time when prescription policy is under ever closer scrutiny. Since alteration in the incidence of insomnia and anxiety in medical patients in the intervening ten years is unlikely, the reduced use of psychotropic drugs to treat these minor complaints as a matter of routine and the marked fall in inappropriate concomitant therapy indicate a hopeful trend towards more rational drug use. This was achieved without fiscal control and was presumably the result of improved education. Further rationalization of prescribing habits may be achieved by self-audit within the profession aided by the activity of drug and therapeutic committees and their extension to general practice. 


\section{References}

BARRETT, C.W., WILSON, A.R. \& BAKER, J.A. (1982). Formularies. In Clinical Pharmacy and Hospital Drug Management, Lawson, D.H. and Richard, R.M.E. (eds), Chapman and Hall: London.

COMMITTEE ON THE REVIEW OF MEDICINES. (1979). Recommendations on barbiturate preparations. British Medical Journal, 2, 719.

GREENBLATT, D.J., SHADER, R.I., DIVOLL, M. \& HARMATZ, J.S. (1981). Benzodiazepines: A summary of pharmacokinetic properties. British Journal of Clinical Pharmacology, 11, $11 \mathrm{~S}$.

GREENBLATT, D.J. \& SHADER, R.I. (1974). In Benzodiazepines in Clinical Practice. Raven Press: New York.
JICK, H., MIETTINEN, O.S., SHAPIRO, S., LEWIS, G.P., SISKIND, V. \& STONE, D. (1970). Comprehensive drug surveillance. Journal of the American Medical Association, 213. 1455.

KESSON, C.M., GRAY, J.M.B. \& LAWSON, D.H. (1976). Benzodiazepine drugs in general medical patients. British Medical Journal, 1, 680.

RICKELS, K. (1981). Are benzodiazepines overused and abused? British Journal of Clinical Pharmacology, 11, 71S.

THOMPSON, T.L., MORAN, M.G. \& NIES, A.S. (1983). Psychotropic drug use in the elderly. New England Journal of Medicine, 308, 134; 194. 